\title{
Erratum to: Report on the 3rd Ottawa International Conference on Neuromuscular Biology, Disease and Therapy - September 24-26, 2015, Ottawa, Canada
}

Jodi Warman Chardon, Bernard J. Jasmin, Rashmi Kothary and Robin J. Parks

Pre-press 22 November 2021

[Journal of Neuromuscular Diseases, 3(3) (2016), 431-442, 10.3233/JND-169001]

https://content.iospress.com/articles/journal-of-neuromuscular-diseases/jnd169001

When this article was first published the first author's first name and last name were incorrectly indexed as "JW Chardon". This has been corrected to "J. Warman-Chardon" in the XML files of the revised online version of the article (DOI: 10.3233/JND-169001). 